\title{
Developing Intestine Is Injured during Absorption of Oleic Acid but Not Its Ethyl Ester
}

Otto R. Velasquez, * Allen R. Place, ${ }^{5}$ Patrick Tso, * and Karen D. Crissinger *

Departments of *Physiology and ${ }^{\ddagger}$ Pediatrics, Louisiana State University Medical Center, Shreveport, Louisiana 71130 ; and ${ }^{\S}$ Center of Marine Biotechnology, University of Maryland, Baltimore, Maryland 21202

\section{Abstract}

Although lipids are essential nutrients in the mammalian diet, we have shown that fatty acids are injurious to epithelial cells of developing piglet intestine during luminal perfusion. Furthermore, the intestine of young animals sustains greater injury than that of older piglets. In an effort to understand the mechanism for this developmental injury, we investigated whether changes in the chemical configuration of oleic acid would alter this damage. Mucosal permeability, as quantitated by the plasma-to-lumen clearance of ${ }^{\mathbf{5 1}}$ chromium EDTA, was evaluated during luminal perfusion with oleic acid as compared with its ethyl (ethyl oleate) and glyceryl (glycerol-1-mono-oleate) esters, solubilized with taurocholic acid, in jejunum of 1-d-, 3-d-, 2-wk-, and 1-mo-old piglets. ${ }^{51}$ Chromium EDTA clearance increased significantly during oleic acid and glycerol-1mono-oleate perfusion, but did not increase during perfusion with ethyl oleate or saline. This result was not secondary to failure of absorption of ethyl oleate, as $\left[{ }^{14} \mathrm{Cloleic}\right.$ acid and ethyl $\left[1-{ }^{14} \mathrm{C}\right]$ oleate were absorbed to a similar extent. Furthermore, developing intestine was able to remove the ethyl group and then re-esterify the fatty acid to form triacylglycerol. These studies indicate that oleic acid-induced mucosal injury can be abolished when the carboxylic group of the fatty acid is esterified with an ethyl, but not a glycerol, group. Since the ethyl ester is also absorbed and metabolized similarly to the free fatty acid, this may provide a means of supplying long-chain fatty acids to developing intestine without causing mucosal damage. (J. Clin. Invest. 1994. 93:479-485.) Key words: necrotizing enterocolitis $\bullet$ mucosal permeability $\bullet$ fatty acids $\bullet$ lipid absorption $\bullet$ infant nutrition

\section{Introduction}

Numerous in vivo and in vitro studies have demonstrated the cytotoxic effects of fatty acids on intestinal cells of adult animals (1-4). Perfusion or incubation of intestinal tissue with fatty acids solubilized with bile salts produces significant cell damage that is not seen with bile salts alone $(2,3)$. This lipid-

Portions of this work were presented at the meeting of the American Gastroenterological Association, May 10-13, 1992, San Francisco, CA. Address correspondence to Dr. Karen D. Crissinger, Department of Pediatrics, Louisiana State University Medical Center, P.O. Box 33932, Shreveport, LA 71130.

Received for publication 6 May 1993 and in revised form 26 August 1993.

J. Clin. Invest.

(c) The American Society for Clinical Investigation, Inc.

$0021-9738 / 94 / 02 / 0479 / 07 \$ 2.00$

Volume 93, February 1994, 479-485 induced injury increases proportionally with the concentration (2) and with the carbon chain length (3) of the fatty acid.

In developing piglet intestine we have demonstrated that mucosal injury induced by dietary fatty acids is also dependent on the fatty acid concentration and carbon chain length $(5,6)$. In general, medium-chain fatty acids (MCFA) ${ }^{1}$ appear to be less injurious to the intestinal mucosa of developing animals compared to long-chain fatty acids (LCFA) at concentrations that may be present postprandially in the intestinal lumen. We have also shown that the sensitivity of the intestinal mucosa to the injurious effects of fatty acids changes as a function of age such that the intestine of 1 -d-old piglets is more prone to injury compared to that of 1-mo-old animals (5).

In addition to the low cytotoxicity of medium-chain fatty acids in intestinal mucosa of developing animals, MCFA are digested and absorbed efficiently from the gastrointestinal tract without the need for bile salt solubilization, providing a readily available source of energy for the newborn (7). This is particularly important in the neonatal period, when pancreatic and biliary functions are immature $(8,9)$. However, dietary MCFA cannot be transformed into essential LCFA in the body (10). Mammals lack the enzymes to transform MCFA into LCFA of the $n-6$ and $n-3$ series, which are necessary for the synthesis of complex structural lipids in biological membranes and are precursors for eicosanoid compounds (11). The LCFA considered essential in human nutrition are the 18-carbon chain $n-6$ linoleic acid (18:2, n-6) and its derivatives, in particular arachidonic acid (20:4, $n-6)$; and the $n-3$ fatty acids linolenic acid (18:3, $n-3)$ and its longer chain, more polyunsaturated derivatives, eicosapentaenoic acid (20:5, $n-3)$ and docosahexaenoic acid (22:6, $n-3)$, as have been identified ( 10 , 12). Therefore, these fatty acids must be provided in the infant diet for normal growth and development.

Previous work from our laboratory has shown that oleic acid, a common dietary long-chain fatty acid, induces increases in mucosal permeability in developing piglet intestine. These changes in permeability are associated with mucosal injury observed with light and electron microscopy (5). Moreover, we demonstrated that the oleic acid-induced injury is similar to that observed with the essential fatty acids linoleic and linolenic acids (6).

There are studies that suggest that the cytotoxicity of longchain fatty acids may be related to the presence of a free carboxyl group in the lipid molecule. Zhu et al. have shown that esterification of oleic acid and linoleic acid with a methyl group significantly decreases the cytotoxic effect of these LCFA on

1. Abbreviations used in this paper: $\mathrm{CE}$, cholesterol ester; DG, diglyceride; EO, ethyl oleate; LCFA, long chain fatty acids; MCFA, medium chain fatty acids; MG, monoglyceride; $n$, omega; PL, phospholipid; PUFA, polyunsaturated fatty acids; TG, triacylglycerol. 
Ehrlich ascites carcinoma cells in mice (13). They concluded that part of the cytotoxicity of LCFA can be attributed to the existence of a free carboxylic group. Similarly, Raz and Livne demonstrated that methyl esters of oleic, linoleic, and linolenic acids do not show the hemolytic effect observed with increasing concentrations of the unesterified (free) fatty acids (14). In this study we investigated whether the injurious effects of oleic acid on intestinal mucosa of developing piglets are influenced by changes in the chemical configuration of the carboxylic group of the fatty acid. Our objective was to gain a better understanding of the pathophysiology of lipid-induced injury in developing intestine. In the course of this investigation we observed that ethyl oleate was harmless to the intestinal mucosa. This intriguing observation raised the question of whether the intestinal absorption and metabolism of ethyl oleate was comparable to those of the free fatty acid. This question was addressed by studying absorption of $\left[{ }^{14} \mathrm{C}\right]$ oleic acid compared to ethyl $\left[1-{ }^{14} \mathrm{C}\right]$ oleate. The results of this study provide insight into the mechanisms of mucosal injury induced by dietary fatty acids and suggest that ethyl esters of LCFA may be an alternative means of supplying these important nutrients to immature intestine.

\section{Methods}

\section{Intestinal permeability studies}

Animal preparation. 20 Hampshire/Yorkshire piglets of either sex were randomly selected among groups of 1-d-old, never-nursed $(1-7 \mathrm{~h}$ of age; $1.51 \pm 0.08 \mathrm{~kg} ; n=5) ; 3$-d-old, fasted for $18 \mathrm{~h}(2.2 \pm 0.12 \mathrm{~kg} ; n$ $=5)$; 2-wk-old, fasted for $24 \mathrm{~h}(4.27 \pm 0.42 \mathrm{~kg} ; n=5)$; and 1-mo-old, fasted for $24 \mathrm{~h}(7.38 \pm 0.63 \mathrm{~kg} ; n=5)$. After intramuscular injection of ketamine hydrochloride $(20 \mathrm{mg} / \mathrm{kg})$ and xylazine $(2 \mathrm{mg} / \mathrm{kg})$, the animals were anesthetized with pentobarbital sodium $(15 \mathrm{mg} / \mathrm{kg})$ via an ear vein. Maintenance doses of pentobarbital $(5 \mathrm{mg} / \mathrm{kg}$ ) were given intravenously as needed during the experiment.

Immediately following anesthetic induction, the animals were artificially ventilated via a tracheostomy with an intermediate ventilator (Harvard Apparatus, South Natick, MA) at a tidal volume and respiratory rate to maintain normal arterial blood gases and $\mathrm{pH}(\mathrm{pH} / \mathrm{blood}$ gas analyzer; Instrumentation Laboratory, Lexington, MA). Polyethylene cannulas were inserted into the right external jugular vein, for administration of pentobarbital and fluid for hydration, and into the left carotid artery to monitor systemic arterial pressure (physiologic recorder; Grass Instrument Co., Quincy, MA) and to withdraw blood samples. Body temperature was maintained at $\sim 37^{\circ} \mathrm{C}$ with a heating pad and an infrared heating lamp.

The abdomen was opened through a midline incision and the renal vessels were ligated to prevent excretion of ${ }^{51} \mathrm{Cr}$-labeled EDTA (New England Nuclear, Boston, MA), which was injected later. Four 10-cm loops of jejunum were isolated and cannulated at both proximal and distal ends with polyethylene tubing, after which the intestinal and abdominal contents were irrigated with normal saline and covered with plastic wrap to prevent evaporative water loss.

Preparations of lipid solutions. 5-mM solutions of oleic acid, glycerol 1-mono-oleate, and ethyl oleate were prepared. The lipids were solubilized with $10 \mathrm{mM}$ taurocholic acid (sodium salt) and emulsified with an ultrasonic homogenizer for $10 \mathrm{~min}$ (Labsonic 2000; Ultrasonic Power, Freeport, IL). All solutions were iso-osmolar ( $300 \mathrm{mosM})$, the $\mathrm{pH}$ was adjusted to $\sim 7.4$ with $1 \mathrm{~N} \mathrm{NaOH}$, and they were maintained in a waterbath at $37^{\circ} \mathrm{C}$. All the chemical compounds were obtained from Sigma Chemical Co. (St. Louis, MO) at the highest purity available.

Experimental protocol. After surgery, the cannulated loops were perfused with warm normal saline via a Gilson minipuls peristaltic pump at a rate of $1 \mathrm{ml} / \mathrm{min}$ to achieve a constant flow. ${ }^{51} \mathrm{Cr}$-labeled EDTA was then injected intravenously, such that there were at least
$25,000 \mathrm{cpm} / \mathrm{ml}(100-150 \mu \mathrm{Ci} / \mathrm{kg}) .10 \mathrm{~min}$ were allowed for tissue equilibration of the labeled EDTA. After the equilibration period, the four intestinal loops were initially perfused with normal saline during 20 min (control period). After this control period, the different fatty acid solutions in each experimental group were perfused simultaneously in different loops for $20 \mathrm{~min}$ (lipid period). Subsequently, the perfusate was changed to normal saline for 60 additional min (recovery period). Luminal perfusate from the effluent cannula $(4 \mathrm{ml})$ was collected at 10 -min intervals during the experiment to monitor ${ }^{51} \mathrm{Cr}$-labeled EDTA clearance. $1 \mathrm{ml}$ of blood (centrifuged to obtain $0.5 \mathrm{ml}$ of plasma) was removed every $20 \mathrm{~min}$ to monitor plasma ${ }^{51} \mathrm{Cr}$-labeled EDTA radioactivity. The blood was replaced with an equivalent volume of 6\% Dextran 70 (Sigma Chemical Co.). ${ }^{51} \mathrm{Cr}$-labeled EDTA activity in plasma and in 4-ml aliquots of perfusate for each $10 \mathrm{~min}$, was measured in a CompuGamma spectrometer (model 1282; LKB Instruments, Inc., Gaithersburg, MD).

At the end of the experiment, the animal was killed with an overdose of pentobarbital $(60 \mathrm{mg} / \mathrm{kg})$, and each loop of intestine was removed, rinsed with normal saline, and weighed.

Calculation of ${ }^{51} \mathrm{Cr}$-labeled EDTA clearance. The plasma-to-lumen clearance of ${ }^{51} \mathrm{Cr}$-labeled EDTA was calculated as follows:

Clearance $=\frac{c p m_{\mathrm{p}} \times p r \times 100}{c p m_{\mathrm{pl}} \times w t}$

where clearance is given in $\mathrm{ml} / \mathrm{min}$ per $100 \mathrm{~g}, c p m_{\mathrm{p}}$ is cpm per $\mathrm{ml}$ of perfusate, $p r$ is the perfusion rate, $c p m_{\mathrm{pl}}$ is $\mathrm{cpm}$ per $\mathrm{ml}$ of plasma, and $w t$ is weight of the intestinal segment in grams.

Data analysis. All values for ${ }^{51} \mathrm{Cr}$-labeled EDTA clearance are expressed as mean \pm SEM. A three-factor ANOVA, followed by Duncan multiple range post hoc testing if the ANOVA revealed differences among groups, was used to evaluate differences among age groups, fatty acid solutions, and experimental periods (SAS Institute Inc., Cary, NC). Differences were considered significant at $P<0.05$.

\section{Absorption studies}

Animal preparation. 21 piglets of either sex were divided into groups of 1-d-old, never nursed ( $n=14)$, and 2-wk-old, fasted for $24 \mathrm{~h}(n=7)$ subjects. These animals were anesthetized and handled as described in the previous section, with the exception that the renal pedicles were left intact.

Preparation of lipid solutions. Solutions of oleic acid and ethyl oleate in normal saline at a concentration of $5 \mathrm{mM}$ were prepared and labeled with $\left[1-{ }^{14} \mathrm{C}\right]$ oleic acid $(20 \mathrm{nCi} / \mathrm{micromol})$, and ethyl $\left[1-{ }^{14} \mathrm{C}\right]-$ oleate ( $20 \mathrm{nCi} /$ micromol), respectively. $10 \mathrm{mM}$ taurocholic acid (sodium salt) was added and the solutions were emulsified for $10 \mathrm{~min}$ with an ultrasonic homogenizer. The $\mathrm{pH}$ was adjusted to $\sim 7.4$ with $1 \mathrm{~N}$ $\mathrm{NaOH}$

$\left[1-{ }^{14} \mathrm{C}\right]$ Oleic acid was obtained from New England Nuclear Research Products (Boston, MA) and had a purity of $99 \%$. The ethyl $\left[1-{ }^{14} \mathrm{C}\right]$ oleate was synthesized using $p$-toluenesulfonic acid-catalyzed esterification (15). The ethyl $\left[1-{ }^{14} \mathrm{C}\right]$ oleate was purified by chromatography on tapered preparative silica G plates (Analtech Inc., Newark, $\mathrm{DE})$ using hexane/diethyl ether $(85: 15, \mathrm{vol} / \mathrm{vol})$, and detected by radiometric scanning after scraping the silica and eluting with hexane containing 2\% diethyl ether. From radiometric scanning of the TLC plate using a Vanguard (model 2001; Digital Diagnostic Corp., Hamden, CT), the radiopurity was $98.7 \%$.

Experimental protocol. After emulsification, reference samples of the lipid solutions from the top, middle, and bottom of the container were obtained. Radioactivity was measured in these samples to ensure a homogeneous distribution of the labeling molecule. Then a volume adequate to fill the intestinal loops without causing distention $(2 \mathrm{ml}$ in 1-d-old, and $5 \mathrm{ml}$ in 2-wk-old) was placed into different loops for $1 \mathrm{~h}$. 12 to 16 loops per lipid solution per age group were used for statistical analysis. In seven 1-d-old piglets, the solutions were left in the loops for up to $3 \mathrm{~h}$ and loops were sequentially excised every $\mathrm{h}$ to determine progressive absorption and intestinal metabolism of the lipid.

At the end of the lipid infusion, the jejunal loops were carefully 
excised and placed over ice. The remaining volume inside the loop was collected in separate conical test tubes. Each loop was then rinsed thoroughly with three washes of a cold solution of $10 \mathrm{mM}$ taurocholic acid and the washes were collected and combined in separate conical tubes. All the volumes were recorded. The samples were mixed with a vortex shaker and an aliquot $(50 \mu \mathrm{l})$ from each sample was taken for radioactivity determination. Lipid absorption was determined as follows:

absorptive index $=$

$100 \%$ of infusate $\mathrm{dpm}-\%$ of total $\mathrm{dpm}$ recovered in lumen.

Lipids in the effusate collected from ethyl $\left[1-{ }^{14} \mathrm{C}\right]$ oleate-infused loops were extracted by the method of Blankenhorn and Ahrens (16) and were analyzed by TLC. The intestinal segments were then opened longitudinally, the mucosa separated via blunt dissection, and placed in separate Erlenmeyer flasks containing $50 \mathrm{ml}$ of chloroform/methanol 2:1 for lipid extraction (17). After $24 \mathrm{~h}$, an aliquot $(0.5 \mathrm{ml})$ from each flask was taken and blown down under nitrogen for radioactivity determination. Lipid transport was calculated as follows:

transport index $=$

$\frac{\text { absorptive index }-\% \text { of total dpm recovered in mucosa }}{\text { absorptive index }} \times 100$

Lipids from the jejunal mucosa of 1-d-old piglets extracted with chloroform/methanol 2:1, were also used to determine the distribution of radioactivity in different lipid classes as analyzed by TLC.

Radioactivity determination. The samples were mixed thoroughly with $10 \mathrm{ml}$ of an aqueous miscible scintillation cocktail (Poly-Fluor; Packard Instrument, Downers Grove, IL) and then counted for $10 \mathrm{~min}$ in a liquid scintillation spectrophotometer (model 1209 Rackbeta; LKB Instruments, Inc.).

$T L C$ analysis. The TLC analysis was performed using Silica Gel G plates $(20 \times 20 \mathrm{~cm})$, which had been activated at $120^{\circ} \mathrm{C}$ for $30 \mathrm{~min}$. The solvent system used was composed of petroleum ether/anhydrous diethyl ether/glacial acetic acid (105:21:0.6, vol/vol/vol). The lipids were visualized after staining with iodine vapor and were identified by co-migrating lipid standards (18). The appropriate lipid spots were then scraped into scintillation vials, and $10 \mathrm{ml}$ of scintillation cocktail was added for radioactivity determination. TLC plates used for photography were stained with $25 \%$ phosphomolybdic acid in methanol.

Data analysis. All values are expressed as means \pm SEM. A threefactor ANOVA, followed by Duncan multiple-range post hoc testing (SAS Institute, Inc., Cary, NC) was used to evaluate differences among age groups, lipid solutions, and time. Differences were considered significant at $P<0.05$.

\section{Results}

\section{Permeability studies}

Perfusion of developing piglet jejunum with $5 \mathrm{mM}$ oleic acid produced significant increases in mucosal permeability in all age groups (Fig. $1 A$ ). This response was more attenuated in 1-mo-old piglets compared to younger animals. Mucosal permeability returned to near baseline values after $1 \mathrm{~h}$ of luminal perfusion with normal saline such that at the end of the experiment these values were not significantly different than control at any age.

A similar response was observed in developing intestine after perfusion with glycerol 1-mono-oleate (Fig. $1 B$ ). Clearance values also returned to near control values at the end of the recovery period. In contrast, after luminal perfusion with ethyl oleate no significant alterations of EDTA clearance were observed at any age during any time period (Fig. $1 C$ ).

When the permeability values obtained after perfusion with the 3 lipids were compared in 1-d-old and 1-mo-old animals, it
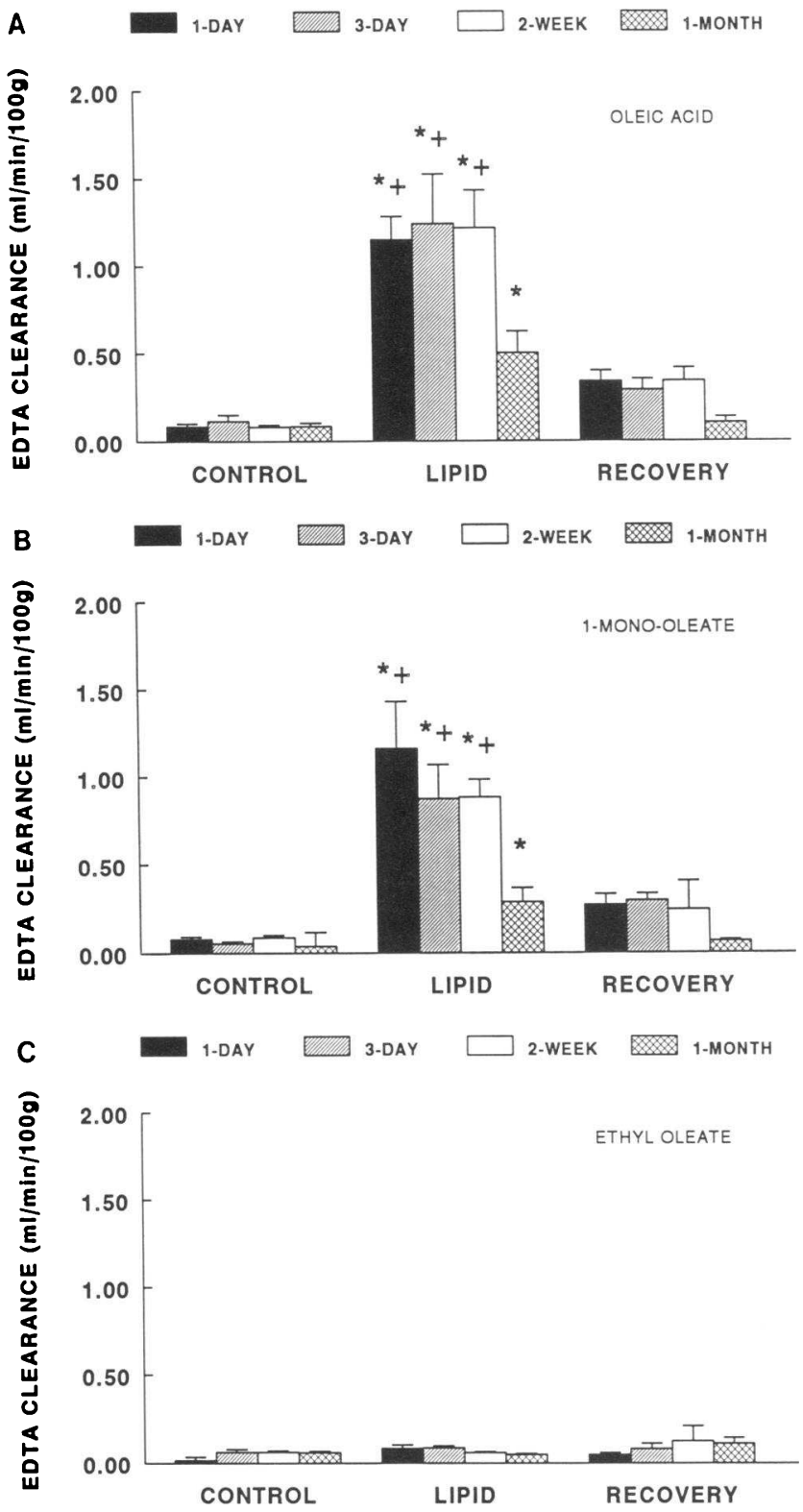

Figure 1. Jejunal ${ }^{51} \mathrm{Cr}$-labeled EDTA clearance (mean \pm SEM) after luminal perfusion with 20 min of normal saline (CONTROL), followed by $20 \mathrm{~min}$ of $5-\mathrm{mM}$ solutions of either oleic acid, glycerol 1mono-oleate, or ethyl oleate, each solubilized with $10 \mathrm{mM}$ taurocholic acid (LIPID), followed by 60 min of normal saline (RECOVERY). Values were obtained at the end of each experimental period in 1-d-, 3-d-, 2-wk-, and 1-mo-old piglets ( $n=5$ animals per age group). ${ }^{*}$ indicates $P<0.05$ vs. control within a lipid solution. + indicates $P$ $<0.05$ vs. 1 -mo-old animals within an experimental time period.

was clear that only oleic acid and glycerol 1-mono-oleate produced significant increases in mucosal permeability in both age groups. Moreover, the mucosal injury observed after perfusion with oleic acid and glycerol 1-mono-oleate in 1-d-old piglets was significantly higher than that observed in 1-mo-old animals (Fig. 2).

\section{Absorption studies}

Ethyl oleate is more hydrophobic than oleic acid or glycerol 1-mono-oleate. Under the experimental conditions used in this study it is possible that the reduced effects of ethyl oleate on mucosal permeability may be due to decreased micellar solubi- 


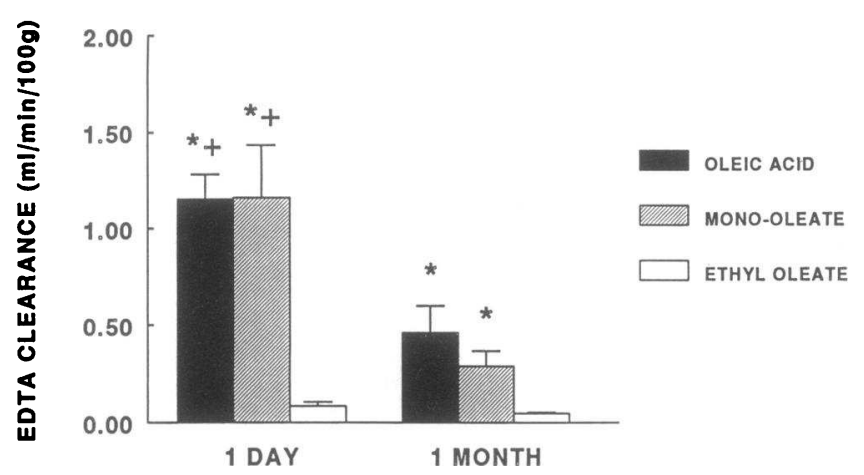

Figure 2. Comparison of EDTA clearance (mean \pm SEM) in jejunal loops luminally perfused for $20 \mathrm{~min}$ with $5 \mathrm{mM}$ oleic acid, glycerol 1 -mono-oleate, or ethyl oleate, each solubilized with $10 \mathrm{mM}$ taurocholic acid in 1-d-old and 1-mo-old animals ( $n=5$ animals per age group). *indicates $P<0.05$ vs. ethyl oleate within an age group. + indicates $P<0.05$ vs. 1-mo-old within a lipid solution group.

lization and, therefore, reduced mucosal contact and absorption of the ethyl ester compared to the free fatty acid. To determine whether the physicochemical characteristics of ethyl oleate were responsible for its attenuated effect, we compared the intestinal absorption of ethyl $\left[{ }^{14} \mathrm{C}\right]$ oleate and oleic acid in 1-d-old and 2-wk-old animals.

Fig. 3 shows the absorption rate of ethyl $\left[1-{ }^{14} \mathrm{C}\right]$ oleate and $\left[1-{ }^{14} \mathrm{C}\right]$ oleic acid in developing piglet intestine. Approximately $75 \%$ of the ethyl $\left[1-{ }^{14} \mathrm{C}\right]$ oleate was absorbed from the lumen after $1 \mathrm{~h}$ and no difference was observed as a function of age. TLC analysis of the effusate collected further supported the high absorptive capability of developing intestine (Fig. 4). Most of the radioactive lipid infused was absorbed and only $\sim 3 \%$ of the lipid present in the effusate was in the ethyl ester form. However, it could not be determined from the TLC analysis if ethyl $\left[1-{ }^{14} \mathrm{C}\right]$ oleate was taken up intact or was hydrolyzed before uptake by the intestinal mucosa. A slightly higher uptake of $\left[1-{ }^{14} \mathrm{C}\right]$ oleic acid than ethyl $\left[1-{ }^{14} \mathrm{C}\right]$ oleate was observed in both age groups. This difference was statistically significant for the 1-d-old piglets (Fig. 3).

To ascertain the proportion of lipid that had actually been

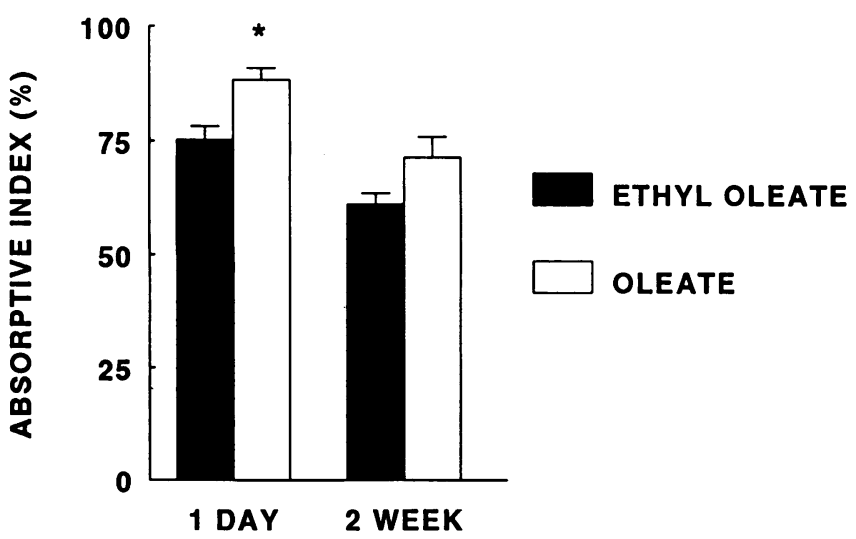

Figure 3. Percentage of total lipid absorbed (mean \pm SEM) after placement of ethyl $\left[1-{ }^{14} \mathrm{C}\right]$ oleate or $\left[1-{ }^{14} \mathrm{C}\right]$ oleic acid in jejunal loops for $1 \mathrm{~h}$ in 1 -d-old and 2 -wk-old piglets ( $n=7$ animals per age group). *indicates $P<0.05$ vs. ethyl oleate within an age group.

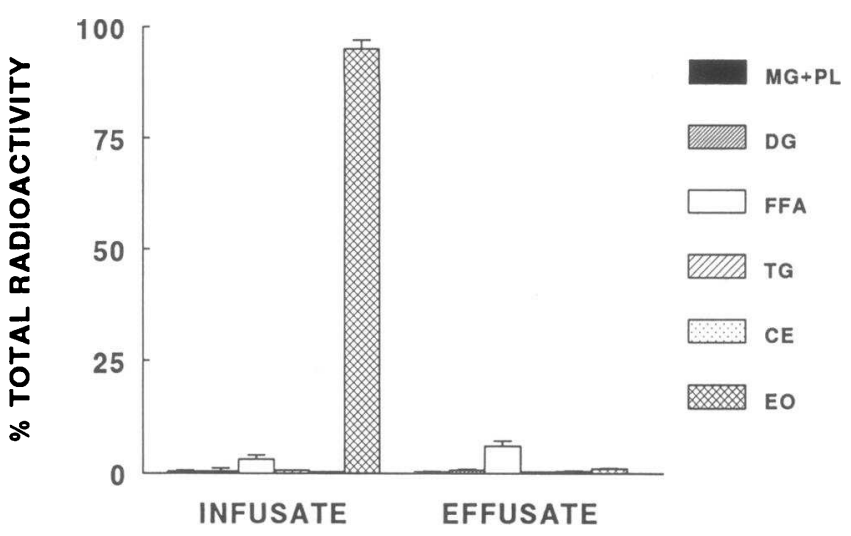

Figure 4. Distribution of ${ }^{14} \mathrm{C}$ radioactivity (mean \pm SEM) in the lipid extracted from the ethyl $\left[1-{ }^{14} \mathrm{C}\right]$ oleate solution placed into the lumen and from the effusate collected after $1 \mathrm{~h}$ in 1-d-old piglets $(n=3$ animals with 4 intestinal segments per animal).

transported out of the intestinal mucosa after $1 \mathrm{~h}$, the amount of radioactive lipid present in the mucosa was determined and the transport index for each lipid was calculated (Fig. 5). Approximately $65 \%$ of the total $\left[1-{ }^{14} \mathrm{C}\right]$ oleic acid placed into the lumen was transported from the intestinal mucosa at the end of the first $h$. This percentage was similar in both age groups. The proportion of ethyl $\left[1-{ }^{14} \mathrm{C}\right]$ oleate transported was similar to that of $\left[1-{ }^{14} \mathrm{C}\right]$ oleic acid in 2-wk-old animals. Interestingly, a significantly lower amount of ethyl $\left[1-{ }^{14} \mathrm{C}\right]$ oleate compared to $\left[1-{ }^{14} \mathrm{C}\right]$ oleic acid was transported out of the mucosa after $1 \mathrm{~h}$ in 1-d-old piglets. This suggested that although a significant proportion of ethyl oleate was absorbed from the lumen in 1-d-old jejunum, there was a decreased ability to metabolize and/or transport the absorbed ethyl ester out of the intestinal mucosa at this age.

To investigate whether this reduced capability to handle ethyl oleate improved with time, the radioactive lipids were placed into separate jejunal loops of 1-d-old piglets for $3 \mathrm{~h}$. Fig. 6 shows that the absorption of ethyl $\left[1-{ }^{14} \mathrm{C}\right]$ oleate was essentially complete by the second infusion $\mathrm{h}$. At this time, uptake was close to $100 \%$ and the difference in absorption rate be-

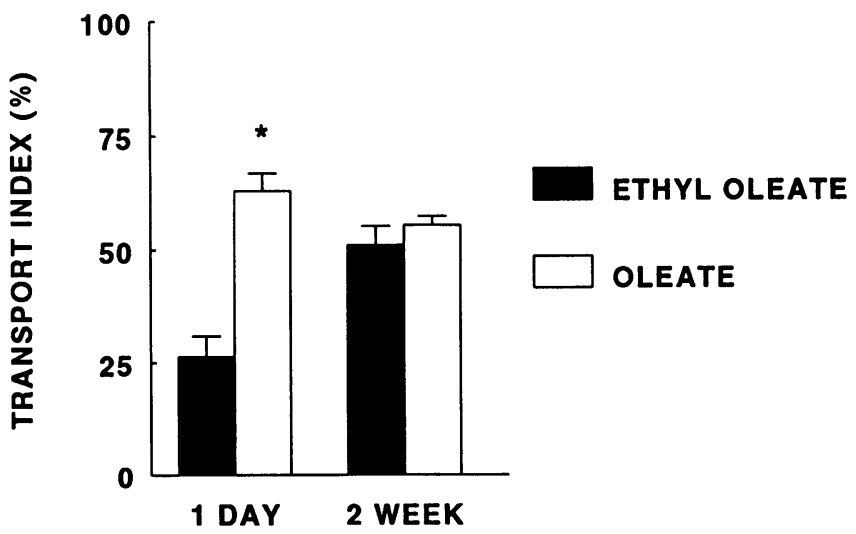

Figure 5. Lipid transport index (mean \pm SEM) expressed as $\%$ of total lipid (ethyl $\left[1-{ }^{14} \mathrm{C}\right]$ oleate or $\left[1-{ }^{14} \mathrm{C}\right]$ oleic acid) placed for $1 \mathrm{~h}$ into jejunal loops of 1-d-old and 2-wk-old piglets ( $n=7$ animals per age group). *indicates $P<0.05$ vs. ethyl oleate within an age group. 


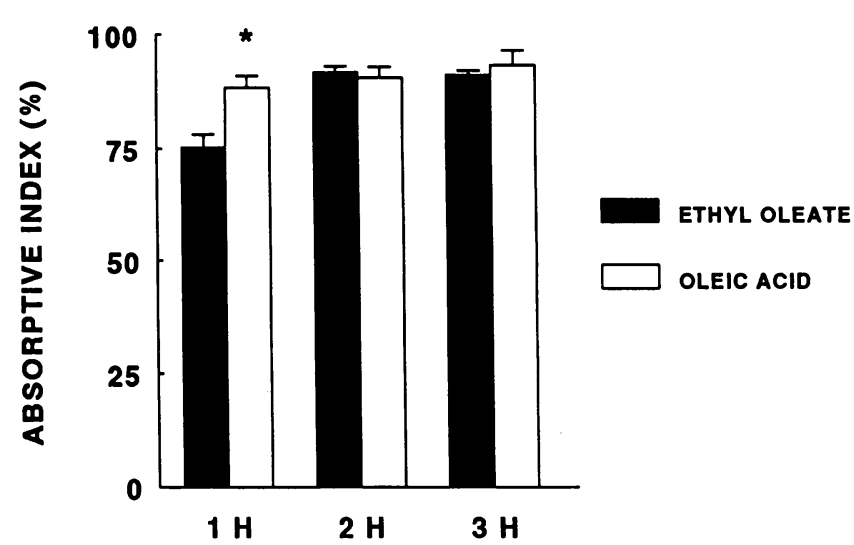

Figure 6. Absorption rate (mean $\pm \mathrm{SEM}$ ) of ethyl $\left[1-{ }^{14} \mathrm{C}\right]$ oleate or $\left[1-{ }^{14} \mathrm{C}\right]$ oleic acid after placement into the jejunum for 1,2 , or $3 \mathrm{~h}$ in 1-d-old piglets ( $n=7$ animals). *indicates $P<0.05$ vs. ethyl oleate within a time period.

tween ethyl $\left[1-{ }^{14} \mathrm{C}\right]$ oleate and $\left[1-{ }^{14} \mathrm{C}\right]$ oleic acid previously observed was abolished. When the transport index was determined, a progressive increase in transport with time for both lipids was observed (Fig. 7). The proportion of $\left[1-{ }^{14} \mathrm{C}\right]$ oleic acid transported was significantly higher than the ethyl $\left[1-{ }^{14} \mathrm{C}\right]-$ oleate transported during the first $2 \mathrm{~h}$ of the experiment. No statistically significant differences between the percentage of either lipid transported out of the mucosa was observed by the third $h$. At this time, the proportion of transported ethyl [1${ }^{14} \mathrm{C}$ ] oleate was significantly higher than the value obtained at $1 \mathrm{~h}$.

The mucosal radioactive lipid was separated into different lipid classes by TLC after $1 \mathrm{~h}$ of lipid infusion. As shown in Fig. 8 , ethyl oleate separated well from triacylglycerols and free fatty acids. The quantitative analysis of this distribution showed that after luminal placement of either ethyl $\left[1-{ }^{14} \mathrm{C}\right]-$ oleate or $\left[1-{ }^{14} \mathrm{C}\right]$ oleic acid, most of the radioactivity was recovered in the triacylglycerol (TG) fraction (Fig. 9). Only a small percentage $(<3 \%)$ of the mucosal lipid was present as ethyl oleate, suggesting that the intestinal mucosa of 1-d-old piglets was able to effectively cleave the ethyl group and re-esterify the resultant lipid into triacylglycerol.

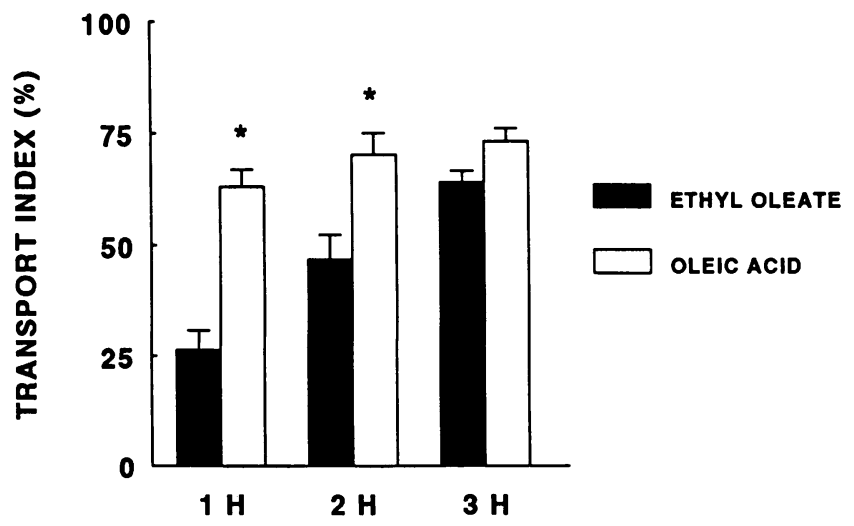

Figure 7. Lipid transport index (mean \pm SEM) expressed as $\%$ of total lipid (ethyl $\left[1-{ }^{14} \mathrm{C}\right]$ oleate or $\left[1-{ }^{14} \mathrm{C}\right]$ oleic acid) placed for 1,2 , or 3 $\mathrm{h}$ into jejunal loops of 1 -d-old piglets $\left(n=7\right.$ animals). ${ }^{*}$ indicates $P$ $<0.05$ vs. ethyl oleate within a time period.

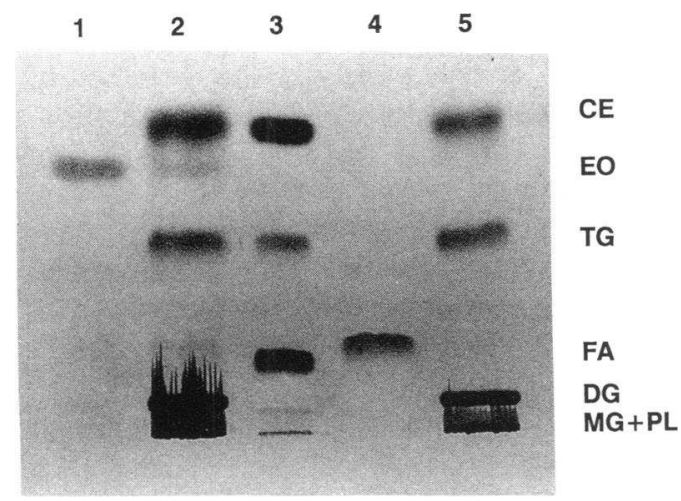

Figure 8. TLC of the lipid extracted from jejunal mucosa of 1-d-old piglets $1 \mathrm{~h}$ after luminal placement of either ethyl $\left[1-{ }^{14} \mathrm{C}\right]$ oleate (column 2 ) or $\left[1-{ }^{14} \mathrm{C}\right]$ oleic acid (column 5 ). Columns 1,3 , and 4 are standards for ethyl oleate, neutral lipid, and oleic acid, respectively.

\section{Discussion}

FFA are highly toxic molecules capable of killing or damaging cells in a variety of tissues including red blood cells $(3,14)$, enterocytes (1-6), several tumor cell lines $(19,20)$, and heart muscle cells (21). Although the mechanism(s) of fatty acid-associated injury is unknown, alterations in the chemical configuration of the hydrophobic group of FFA may lead to changes in their cytotoxic effects. We and others have shown that the injurious effects of FFA on intestinal cells are dependent on the length and, therefore, the hydrophobicity of the carbon chain $(3,6)$. The degree of saturation of the hydrophobic group does not appear to play an important role in determining the toxic effect of fatty acids (6). Information regarding the effect of changes in the configuration of the hydrophilic group on the toxic potential of FFA is sparse. A few studies have evaluated in vitro the cell injury induced by methyl esters of long-chain fatty acids (LCFA), including oleic acid, in red blood cells (14) and tumor cells (13). These experiments showed that the toxic effects of LCFA were abolished after esterification with the methyl group, leading the investigators to conclude that the cytotoxicity of LCFA was dependent on the presence of a free carboxylic group (13).

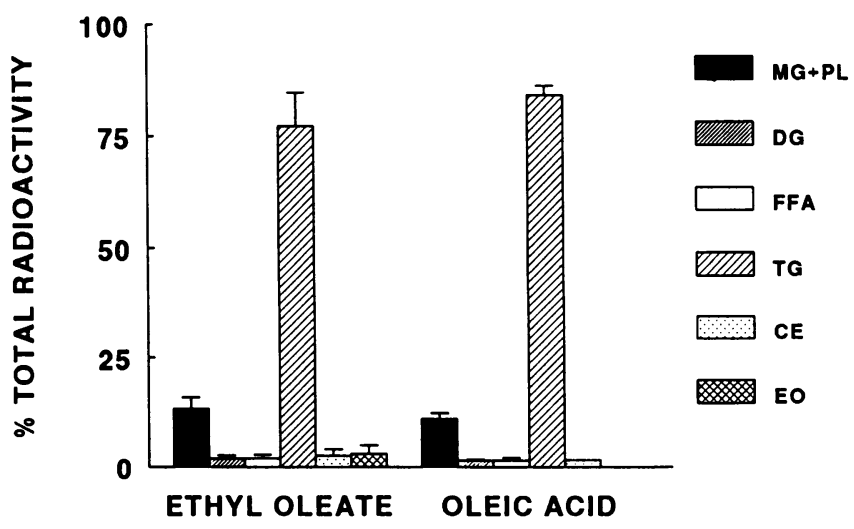

Figure 9. Distribution of mucosal ${ }^{14} \mathrm{C}$ radioactivity (mean \pm SEM) in different lipid classes in 1-d-old piglets ( $n=7$ animals) after placement of ethyl $\left[1-{ }^{14} \mathrm{C}\right]$ oleate or $\left[1-{ }^{14} \mathrm{C}\right]$ oleic acid in jejunal loops for $1 \mathrm{~h}$. 
The objective of the present study was to evaluate whether modifications of the carboxylic moiety by the formation of esters could alter the mucosal injury previously observed $(5)$ in developing piglets after perfusion with oleic acid. Both glycerol 1-mono-oleate and ethyl oleate lack a free carboxylic group, and the major difference between these two compounds relates to the polarity of the ester. The glycerol moiety of the glycerol 1-mono-oleate with two hydroxyl groups is more polar than oleic acid with a single hydroxyl group. Oleic acid is in turn much more polar than the ethyl ester as observed with TLC (Fig. 9). Due to the reduced polarity of the ethyl oleate molecule, its ability to behave as an amphiphile may be impaired, thus lowering its cytotoxicity. Amphiphilic molecules, which possess detergent-like characteristics, may interact with cell membranes and cause alterations ranging from subtle changes in permeability to membrane lysis, according to the concentration of the detergent (22). This direct effect may explain the rapidity of the fatty acid-induced injury observed in this and other studies. The concentration of oleic acid used in this study ( $5 \mathrm{mM}$ ) consistently produced a 20 -fold increase in mucosal permeability (Fig. 1), and has been shown to be associated with cell membrane disruption and cell death observed with light and electron microscopy (5). The mechanism of this amphiphile-induced alteration in cell membranes is unknown but may involve alterations in membrane fluidity and/or membrane breakdown and solubilization into mixed micelles (23).

An alternative explanation for the reduced mucosal injury observed after perfusion with ethyl oleate is suggested by the behavior of this lipid in water. In this study, sodium taurocholate was used to solubilize the lipids in water, and under our experimental conditions ( $\mathrm{pH} \mathrm{7.4,} \mathrm{T} 37^{\circ} \mathrm{C}$ ) oleic acid and glycerol 1-mono-oleate were adequately solubilized. In contrast, ethyl oleate formed a turbid solution. These observations correlated with previous reports by Hofmann (24) that micellar solubilization of solutes with bile salts appears to be influenced by the polarity of the compound such that nonpolar molecules are solubilized less efficiently in an aqueous environment than polar ones. This suggests that the reduced effect of ethyl oleate was secondary to the reduced solubility of this lipid in water, leading to reduced micellar solubilization, reduced mucosal contact, and therefore, reduced mucosal injury. Previous studies in vitro in red blood cells and Caco- 2 cells have shown that stearic acid (C18:0) has a significantly lower lytic potential at $37^{\circ} \mathrm{C}$ compared to other 18 -carbon mono- and polyunsaturated fatty acids $(3)$. This was attributed to the insolubility of the fatty acid secondary to gel formation at this temperature, which is below its critical temperature. To ascertain whether the behavior of ethyl oleate in water was responsible for the lack of injurious effects on intestinal mucosa, we evaluated the ethyl oleate-mucosa interaction by determining the absorption of this lipid and comparing it with the absorption of oleic acid. In 2-wk-old animals $\sim 70 \%$ of the lipid infused was absorbed after $1 \mathrm{~h}$ and no statistically significant differences were observed between the uptake of oleic acid and ethyl oleate (Fig. 3). In 1-d-old piglets the overall absorption rate for both lipids was also high $(\sim 80 \%)$, but the uptake of oleic acid was significantly higher than the uptake of ethyl oleate (Fig. 3). This difference was abolished with time (Fig. 6). These results suggest that mechanisms other than lack of interaction between the ethyl ester and intestinal mucosa may be responsible for the reduced effect of ethyl oleate.

To our knowledge, this is the first report on the absorption of a LCFA and its ethyl ester in developing intestine. Important findings have been described in numerous studies, however, in which the absorption of long-chain polyunsaturated fatty acids (PUFA) in different chemical forms (including FFA, triacylglycerol, and arginine salt) has been compared to the absorption of ethyl esters of PUFA in adult rats (25) and humans (26-29). In general, it has been observed that the absorption of ethyl esters of PUFA is less efficient compared to the other chemical forms, especially FFA. However, it has also been shown that the ability to absorb ethyl esters significantly improves with exposure time (25). The different absorption of ethyl esters of LCFA may be the result of poor hydrolysis by pancreatic lipase (30). It has been suggested that the resistance to hydrolysis could result from the ethyl component of the molecule (26). Studies by Sarda and Desnuelle have reported a lower hydrolysis of ethyl oleate compared to the hydrolysis of triolein (31). Furthermore, it has been shown that the hydrolysis of ethyl oleate is significantly lower than the hydrolysis of the homologous methyl oleate (32). Despite the reduced hydrolysis of ethyl esters of LCFA, this and other studies (33) have reported uptake rates higher than $90 \%$, suggesting that the absorption of the ethyl ester is probably very effective and may take place without prior enzymatic hydrolysis. Once inside the enterocyte, it appears that an intracellular esterase exists that hydrolyzes the ethyl ester, allowing the incorporation of the liberated fatty acid into de novo synthesized triacylglycerols in the cell (Fig. 9). Studies in adult animals have also reported the possible intracellular metabolism of ethyl esters of PUFA. In rats receiving eicosapentaenoic acid and docosahexaenoic acid ethyl esters, no ethyl esters were found either in 4-h lymph samples or in blood (34).

An interesting observation in our study relates to the reduced ability of 1-d-old intestine to transport the absorbed ethyl oleate (Fig. 5). In rats, the ability of small intestine to transport lipid into lymph can be determined by cannulation of a major intestinal lymph duct (35). This allows the calculation of the lymphatic transport index (36). However, in developing piglets lymphatic cannulation is technically very difficult and even though we have been able to cannulate a lymphatic and obtain lymph for a short period of time in 2-wk-old piglets (data not shown), this procedure is impractical in 1-d-olds due to the size and the fragility of the vessels. Another problem is the difficulty in ensuring the complete recovery of intestinal lymph from a certain segment of the gut. Therefore, in our model, transport index was calculated by subtracting the proportion of radioactive lipid present in the mucosa from the proportion of lipid absorbed from the lumen and dividing the result by the absorptive index. This provides lipid transport values that are normalized for the variability in uptake rate that may be observed in different experimental animals. Our results showed that in 1-d-old animals more ethyl oleate was present in the intestinal mucosa after $1 \mathrm{~h}$ compared to oleic acid, indicating a more efficient transport of oleic acid. This phenomenon was not observed in 2-wk-olds (Fig. 5). The reduced ability of 1-day-old intestine to transport the absorbed ethyl oleate was not due to the inability to incorporate the lipid into triacylglycerol, because only a very small amount of ethyl oleate was found in the mucosa at the end of $1 \mathrm{~h}$ (Fig. 9).

The explanation for this phenomenon is unclear. It may be possible that 1-d-old animals use the free fatty acid as an additional metabolic fuel, considering the high metabolic demands of the newborn intestine (37). This would reduce the amount 
of oleic acid in the mucosa and would give a high transport index value. Another possibility is that oleic acid, after causing mucosal epithelial disruption, may reach the interstitium and the circulation through a paracellular route, reducing the amount of the lipid detected in the mucosa. This speculation is not supported by the fact that oleic acid produces similar mucosal injury in 2-wk-old piglets (Fig. 1) and in this age group no difference in the amount of oleic acid and ethyl oleate transported has been found (Fig. 5). Regardless of the mechanism, it is obvious that the initial delay in the transport of ethyl oleate improves significantly with time (Fig. 7). Overall, these results indicate that ethyl oleate is efficiently absorbed, metabolized, and transported out of the mucosa in developing intestine.

Taken together, the results of our study may have important clinical implications in developing intestine. Immaturity or disruption of the developing mucosal barrier may result in clinical disease states to which the newborn is susceptible, such as necrotizing enterocolitis, toxigenic diarrhea, and intestinal allergy. We have shown in this study and others $(5,6)$ that bile acid-solubilized dietary fatty acids, especially the long-chain unsaturated species, in a concentration that may be found postprandially (after pancreatic enzyme digestion of triglycerides in infant formula) are able to disrupt the intestinal mucosal barrier. Because long-chain fatty acids are essential for normal growth and development and must be provided in the diet, it is important to find the means to supply fatty acids to the newborn with minimal alterations of the mucosal barrier, thus decreasing the hazard of intestinal pathology. Our study indicates that esterification of the injurious LCFA oleate with a nonpolar ethyl group abolishes its cytotoxic effects in this experimental setting, using developing piglet intestine. In addition, ethyl oleate is well absorbed and metabolized, indicating that ethyl esters of LCFA may be useful as a source of essential fatty acids to the newborn without concomitantly increasing the risk of mucosal injury. Although we have found no significant differences in injury induced by oleic acid solubilized in bile vs. taurocholic acid (data not shown), investigation of the response to a mixed nutrient meal that has been digested and solubilized by pancreatic enzymes and bile in an intact developing gastrointestinal tract is crucial before applying these experimental findings to the clinical situation.

\section{Acknowledgments}

This work was supported by grants DK-43785 and DK-32288 from the National Institutes of Health.

\section{References}

1. Gaginella, T. S., and S. F. Phillips. 1976. Ricinoleic acid (castor oil) alters intestinal surface structure. Mayo Clin. Proc. 51:6-12.

2. Kvietys, P. R., R. D. Specian, M. B. Grisham, and P. Tso. 1991. Jejunal mucosal injury and restitution: role of hydrolytic products of food digestion. Am. J. Physiol. 261:G384-G391.

3. Lapre, J. A., D. S. M. L. Termont, A. K. Groen, and R. Van der Meer. 1992. Lytic effects of mixed micelles of fatty acids and bile salts. Am. J. Physiol. 263:G333-G337.

4. Morehouse, J. L., R. D. Specian, J. J. Stewart, and R. D. Berg. 1986. Translocation of indigenous bacteria from the gastrointestinal tract of mice after oral ricinoleic acid treatment. Gastroenterology. 91:673-682.

5. Velasquez, O. R., K. Henninger, M. Fowler, P. Tso, and K. D. Crissinger. 1993. Oleic acid-induced mucosal injury in developing piglet intestine. Am. J. Physiol. 264:G576-G582.

6. Velasquez, O. R., P. Tso, and K. D. Crissinger. 1993. Fatty acid-induced injury in developing piglet intestine: effect of degree of saturation and carbon chain length. Pediatr. Res. 33:543-547.
7. Borum, P. R. 1992. Medium-chain triglycerides in formula for preterm neonates: implications for hepatic and extrahepatic metabolism. J. Pediatr. 120:S139-S145.

8. Finley, A. J., and M. Davidson. 1980. Bile acid excretion and patterns of fatty acid absorption in formula-fed premature infants. Pediatrics. 65:132-138.

9. Lebenthal, E., and P. C. Lee. 1980. Development of functional response in human exocrine pancreas. Pediatrics. 66:556-560.

10. Innis, S. M. 1992. Human milk and formula fatty acids. J. Pediatr. 120:S56-S61

11. Hernell, O. 1990. The requirements and utilization of dietary fatty acids in newborn infant. Acta Pediatr. Scand. 365:S20-S27.

12. Connor, W. E., M. Neuringer, and S. Reisbick. 1992. Essential fatty acids: the importance of $n-3$ fatty acids in retina and brain. Nutr. Rev. 50:21-29.

13. Zhu, Y. P., Z. W. Su, and C. H. Li. 1989. Growth-inhibition effects of oleic acid, linoleic acid, and their methyl esters on transplanted tumors in mice. $J$. Natl. Cancer Inst. 81:1302-1306.

14. Raz, A., and A. Livne. 1973. Differential effects of lipids on osmotic fragility of erythrocytes. Biochim. Biophys. Acta. 311:222-229.

15. Place, A. R., and D. D. Roby. 1986. Assimilation and deposition of dietary fatty alcohols in Leach's storm petrel, Oceanodroma leucorhoa. J. Exp. Zool. 240:149-161.

16. Blankenhorn, D. H., and E. H. Ahrens. 1955. Extraction, isolation, and identification of hydrolytic products of triglyceride digestion in man. J. Biol. Chem. 212:69-81.

17. Folch, J., M. Lees, and G. H. Sloan-Stanley. 1957. A simple method for the isolation and purification of total lipid from animal tissue. J. Biol. Chem. 266:497-509.

18. Kritchevsky, D., and M. R. Kirk. 1952. Detection of steroids in paper chromatography. Arch. Biochem. Biophys. 35:346-351.

19. Siegel, I., T. L. Liu, E. Yaghoubzadeh, and T. S. Keskey. 1987. Cytotoxic effects of free fatty acids on ascites tumor cells. J. Natl. Cancer Inst. 78:271-277.

20. Begin, M. E., G. Ells, and D. F. Horrobin. 1988. Polyunsaturated fatty acid-induced cytotoxicity against tumor cells and its relationship to lipid peroxidation. J. Natl. Cancer Inst. 80:188-194.

21. Wenzel, D. C., and T. W. Hale. 1978. Toxicity of free fatty acids for cultured rat heart muscle and endothelioid cells. II. Unsaturated long-chain fatty acids. Toxicology. 11:119-125.

22. Helenius, A., and K. Simons. 1975 . Solubilization of membranes by detergents. Biochim. Biophys. Acta. 415:29-79.

23. Katz, A. M., and F. C. Messineo. 1981. Lipid-membrane interactions and the pathogenesis of ischemic damage in the myocardium. Circ. Res. 48:1-16.

24. Hofmann, A. F. 1963. The function of bile salts in fat absorption. The solvent properties of dilute micellar solutions of conjugated bile salts. Biochem. $J$. 89:57-68.

25. Reicks, M., J. Hoadley, S. Satchithanandam, and K. Morehouse. 1990. Recovery of fish oil-derived fatty acids in lymph of thoracic duct-cannulated Wistar rats. Lipids. 25:6-10.

26. El Boustani, S., C. Colette, L. Monnier, B. Descomps, A. Crastes de Paulet, and F. Mendy. 1987. Enteral absorption in man of eicosapentaenoic acid in different chemical forms. Lipids. 22:711-714.

27. Lawson, L. D., and B. G. Hughes. 1988. Human absorption of fish oil fatty acids as triacylglycerols, free acids, or ethyl esters. Biochem. Biophys. Res. Comm. 152:328-335.

28. Lawson, L. D., and B. G. Hughes. 1988. Absorption of eicosapentaenoic acid and docosahexaenoic acid from fish oil triacylglycerols or fish oil ethyl esters co-ingested with a high fat meal. Biochem. Biophys. Res. Comm. 156:960-963.

29. Nørdoy, A., L. Barstad, W. E. Connor, and L. Hatcher. 1991. Absorption of the $n-3$ eicosapentaenoic and docosahexaenoic acids as ethyl esters and triglycerides by humans. Am. J. Clin. Nutr. 53:1185-1190.

30. Nelson, G. J., and R. G. Ackman. 1988. Absorption and transport of fat in mammals with emphasis on $n-3$ polyunsaturated fatty acids. Lipids. 23:10051014.

31. Sarda, L., and P. Desnuelle. 1958. Action de la lipase pancréatique sur les esters en émulsion. Biochim. Biophys. Acta. 30:513-521.

32. Mattson, F. H., and R. A. Volpenhein. 1969. Relative rates of hydrolysis by rat pancreatic lipase of esters of $\mathrm{C}_{2}-\mathrm{C}_{18}$ fatty acids with $\mathrm{C}_{1}-\mathrm{C}_{18}$ primary $n$-alcohols. J. Lipid Res. 10:271-276.

33. Hamazaki, T., M. Urakaze, M. Makuta, A. Ozawa, Y. Soda, H. Tatsumi, S. Yano, and A. Kumagai. 1987. Intake of different eicosapentaenoic acid-containing lipids and fatty acid pattern of plasma lipids. Lipids. 22:994-998.

34. Carlier, H., A. Bernard, and C. Caselli. 1991. Digestion and absorption of polyunsaturated fatty acids. Reprod. Nutr. Dev. 31:475-500.

35. Bollman, J. L., J. C. Cain, and J. H. Grindlay. 1949. Techniques for the collection of lymph from the liver, small intestine, or thoracic duct of the rat. $J$. Lab. Clin. Med. 33:1349-1352.

36. Bergstedt, S. E., H. Hayashi, D. Kritchevsky, and P. Tso. 1990. A comparison of absorption of glycerol tristearate and glycerol trioleate by rat small intestine. Am. J. Physiol. 259:G386-G393.

37. Velasquez, O. R., D. N. Granger, and K. D. Crissinger. 1992. Intestinal microcirculation in the neonate. Pediatr. Surg. Int. 7:408-414. 